\title{
The pipelined metabolite identification based on MS fragmentation
}

\author{
Miguel Rojas-Cherto ${ }^{*}$, PT Kasper, Peironcely Julio E, T Reijmers, RJ Vreeken, T Hankemeier \\ From 5th German Conference on Cheminformatics: 23. CIC-Workshop \\ Goslar, Germany. 8-10 November 2009
}

Structural characterization and identification of components of complex biological mixtures constitutes one of the central aspects of metabolomics. Metabolite identification is a challenging but essential task in studies of biological samples. Mass spectrometry, because of its high sensitivity and specificity, is widely and successfully used in analysis of biological samples. Identification of metabolites can be in principle achieved using high resolution multistage mass spectrometry (MSn) because it provides a feature rich fingerprint of the precursor structure. However, neither general methodology for the identification nor extensive databases of metabolites with multistage mass spectrometric data are available at the moment. We demonstrate in this poster the feasibility of the strategy for metabolite identification based on analysis of fragmentation trees.

High resolution multi stage MS experiments were performed on LTQ-Orbitrap (Thermo) equipped with Triversa nanoMate (Advion) nanoelectrospray ion source using defined protocol. An in-house developed software, integrating among others: Chemistry Development Kit (CDK) and XCMS libraries, was used for spectral data processing. Resulting fragmentation trees were stored in a local database.

Multi-stage Molecular Formula (MMF) tool, which uses a method to resolve the elemental composition of the compound and fragment ions derived from MSn data using a cyclic constraining process has been developed. The process of elemental formula assignment and fitting within elemental formula paths removes artefacts of the spectra. Background signals, spikes, contaminations, side peaks, etc., are rejected in this stage and are not included in the fragmentation tree. The resulting fragmentation trees are stored in XML format.

Repeatability, reproducibility and robustness of fragmentation tree acquisitions were tested by changing experimental conditions and varying the concentration of the metabolite of interest. An acquisition protocol was established for the reliable and reproducible acquisition of mass spectral trees. It was investigated to which extent the variation of conditions such as fragmentation energy, isolation width etc. did change the fragmentation pattern or topology of hierarchical relations between fragments.

We demonstrate how the developed analytical strategy based on analysis of fragmentation trees can be used to discriminate between metabolite isomers with the same elemental composition and an only slightly different structure, but with a significantly different biological function.

Our results provide firm basis for developing a generic, multi stage mass spectrometry based platform for efficient identification of metabolites.

Published: 4 May 2010

doi:10.1186/1758-2946-2-S1-P53

Cite this article as: Rojas-Cherto et al:: The pipelined metabolite identification based on MS fragmentation. Journal of Cheminformatics 2010 2(Suppl 1):P53. 